OPEN ACCESS

Edited by:

Lu Cai,

University of Louisville, United States

Reviewed by:

Dongfeng Zhang,

Qingdao University, China

Benli Su,

Second Hospital of Dalian Medical

University, China

*Correspondence:

Hongying Jia

jiahongying@sdu.edu.cn

Specialty section: This article was submitted to

Clinical Diabetes,

a section of the journal

Frontiers in Public Health

Received: 28 November 2021 Accepted: 28 December 2021

Published: 28 January 2022

Citation:

Cheng F, Li Y, Zheng H, Tian L and Jia $H$ (2022) Mediating Effect of Body

Mass Index and Dyslipidemia on the Relation of Uric Acid and Type 2 Diabetes: Results From China Health and Retirement Longitudinal Study.

Front. Public Health 9:823739.

doi: 10.3389/fpubh.2021.823739

\section{Mediating Effect of Body Mass Index and Dyslipidemia on the Relation of Uric Acid and Type 2 Diabetes: Results From China Health and Retirement Longitudinal Study}

\author{
Fang Cheng ${ }^{1,2}$, Yanzhi Li ${ }^{1}$, Han Zheng ${ }^{1}$, Lu Tian ${ }^{1}$ and Hongying Jia ${ }^{1,2 *}$ \\ ${ }^{1}$ Department of Epidemiology and Health Statistics, School of Public Health, Cheeloo College of Medicine, Shandong \\ University, Jinan, China, ${ }^{2}$ Center of Evidence-Based Medicine, Institute of Medical Sciences, The Second Hospital, Cheeloo \\ College of Medicine, Shandong University, Jinan, China
}

Objective: This study assessed temporal relationships of serum uric acid (SUA) with blood glucose and determine the mediating effects of body mass index (BMI) and dyslipidemia on the relation of SUA and risk of type 2 diabetes.

Methods: Participants aged $\geq 45$ years were participated in 2011 and followed up until 2015. Cox proportional hazards regression with a robust variance estimator was performed to explore the association of SUA with the risk of diabetes, and crosslagged path analysis was introduced to examine the temporal relationships between SUA and blood glucose. A mediation analysis was finally used to identify the mediating effect of $\mathrm{BMI}$ and dyslipidemia on the relation of SUA and the future risk of diabetes.

Results: A total of 9,020 participants were included with an average age of 58.59 years at baseline in 2011, and $53.6 \%$ of them were women. Linear dose-response relationship was identified by restricted spline cubic analysis between baseline SUA and follow-up blood glucose (the non-linear trend for fasting plasma glucose (FPG): $\beta_{2}=-0.71, p=0.52$; for HbA1c: $\beta_{2}=0.05, p=0.07$; for risk of diabetes: $\beta_{2}=0.12$, $p=0.39$ ). Additionally, compared with the lowest quartiles of SUA, the adjusted risk ratios of diabetes were 1.00 (95\% Cl: 0.82-1.23), 1.08 (95\% Cl: 0.89-1.31), and 1.37 (95\% Cl: 1.11-1.96) for quartile $2-4$ ( $p$-trend < 0.01), respectively. Further additional adjustments for BMl or dyslipidemia, these ratios were not statistically significant. In addition, a unidirectional relationship from baseline SUA to follow-up FPG ( $\rho_{1}=0.24$, $p=0.03$ ) was further confirmed using crosslagged path analysis. After stratifying by genders, the above results were only significant in the women subgroup, and we thus conducted a mediation analysis in women and found that the BMI and dyslipidemia partially mediated the effect of SUA on diabetes with a 23.05 and $18.82 \%$ mediating effect, respectively.

Conclusions: These findings provide strong evidence that hyperuricemia preceded diabetes, and the effect of baseline SUA on follow-up type 2 diabetes was more pronounced among middle-aged and elderly Chinese women, especially in 
postmenopausal women, and this effect is partly mediated by BMI and dyslipidemia at baseline.

Keywords: diabetes, BMI, uric acid, dyslipidemia, mediation effect

\section{INTRODUCTION}

Type 2 diabetes is a metabolic disease characterized by insulin resistance, which affected over 463 million people in 2019, and this number is expected to increase to 578 million in 2,030 and 700 million in 2045 (1). Identifying all potential controllable risk factors for the incidence and development of diabetes is essential for its early screening and prevention.

As the main component of metabolic syndrome, diabetes, hyperglycemia, and hyperlipidemia interconnect and influence each other, forming a complex framework of chronic diseases (2). The link between hyperuricemia and diabetes has been well documented in the previous studies (3-6), some of them demonstrated that for every $1 \mathrm{mg} / \mathrm{dL}$ increase in serum uric acid (SUA) concentration, the risks of type 2 diabetes were increased by $6-11 \%$ (6), and these studies also showed differences between genders. Meanwhile, the epidemiological and clinical evidence supports a strong significant positive association between SUA and obesity in the adult population of China, Japan, India, Pakistan, and Iraq $(7,8)$. About $44 \%$ of diabetes cases are overweight or obese (9), and adults with body mass index $(\mathrm{BMI})>35 \mathrm{~kg} / \mathrm{m}^{2}$ are 20 times as likely to develop type 2 diabetes than those with a BMI between 18.5 and 24.9 $\mathrm{kg} / \mathrm{m}^{2}$. Also, some researchers reported that the relation of SUA and diabetes is largely decreased or eliminated when additional adjusting $\operatorname{BMI}(10,11)$. The possible mechanism is that hyperuricemia can cause obesity by accelerating liver and peripheral fat production (12). In addition, dyslipidemia is a common comorbidity in patients with diabetes (13), and over $70 \%$ of them have one or more lipid abnormalities. Low levels of high-density lipoprotein cholesterol (HDL-C) are often associated with elevated triglyceride levels, the most prevalent form of dyslipidemia in patients with diabetes (14). Studies illustrated that SUA can inhibit the synthesis of adiponectin in adipocytes by reducing the production of nitric oxide in arterial endothelial cells, disrupting the tricarboxylic acid cycle and the oxidation of fatty acid $\beta$, and finally promoting the oxidative activity of cells (15).

Besides, available data suggest that uric acid is not necessarily an antioxidant and, depending on the chemical milieu, may become a prooxidant $(16,17)$. This partly explained the $\mathrm{U}$ - or L-shaped association between SUA and blood glucose reported in previous cross-sectional studies $(18,19)$. What is more, Rodriguez and his colleagues identified that individuals with prediabetes are at a higher risk of developing gout, but once they develop diabetes, their risk drops to a lower level than that of nondiabetic individuals, and diabetes may reduce the future risk of gout through the uricosuric effect of glycosuria or the impaired inflammatory response (20).

To clarify the complex interaction between metabolic syndrome components and formulate reasonable diabetes control measurements, this study collected data from a nationally representative database, the China Health and Retirement Longitudinal Study (CAHRLS), to initially explore whether the uric acid level is independently related to the future risk of diabetes and then introduced a restricted cubic spline function to identify whether there is a non-linear relationship between baseline uric acid and follow-up blood glucose; further used crosslagged path analysis to determine the temporal relation of blood uric acid and blood glucose. Once the temporal relationship is established, we would investigate the mediating effect of BMI and dyslipidemia on the relation of uric acid and risk of diabetes.

\section{MATERIALS AND METHODS Study Population}

The China Health and Retirement Longitudinal Study (CHARLS) takes the mainland of China as the sampling frame, the community (in the city) or village (in urban) as the sampling unit, and uses probability proportionate to size sampling (PPS) as the sampling technology, to investigate the information on health and retirement of middle-aged and elderly people aged 45 or over, with no upper age limit (21). The CHARLS data can be freely downloaded from the official website (http:// charls.pku.edu.cn/index/zh-cn.html). So far, CHARLS yields four waves of data in 2011, 2013, 2015, and 2018 and two blood test data in 2011 and 2015. Information about demographics, biomedical measurements, socioeconomic status, and selfreported health status and functioning was measured by trained health workers (21), and all participants were asked to take venous blood on fasting overnight (22). The CHARLS is a large-scale interdisciplinary research project sponsored by the National Development Institute of Peking University. Ethical approval for all the CHARLS waves, therefore, was granted from the Institutional Review Board (IRB) at Peking University, including anthropometrics (IRB00001052-11015) and biomarker collection (IRB00001052-11014).

In this study, at baseline (2011), a total number of 17,708 respondents completed a face-to-face computer-assisted personal interview, and the exclusion criteria were as follows: (1) without blood samples; (2) missing value of SUA, fasting blood glucose (FPG), hemoglobin A1c (HbA1c), blood urea nitrogen (BUN), estimated glomerular filtration rate (eGFR); (3) with cancer; and (4) diagnosed with type 2 diabetes. A number of 9,431 participants were remained at baseline after exclusion criteria. In 2013 and $2015,8,545$ and 8,323 subjects were successfully followed, and 68 and 762 of them were diagnosed with diabetes, respectively. A number of 9,020 participants were finally followed up, and 795 of them were diagnosed with type 2 diabetes. More details are shown in Figure 1. 


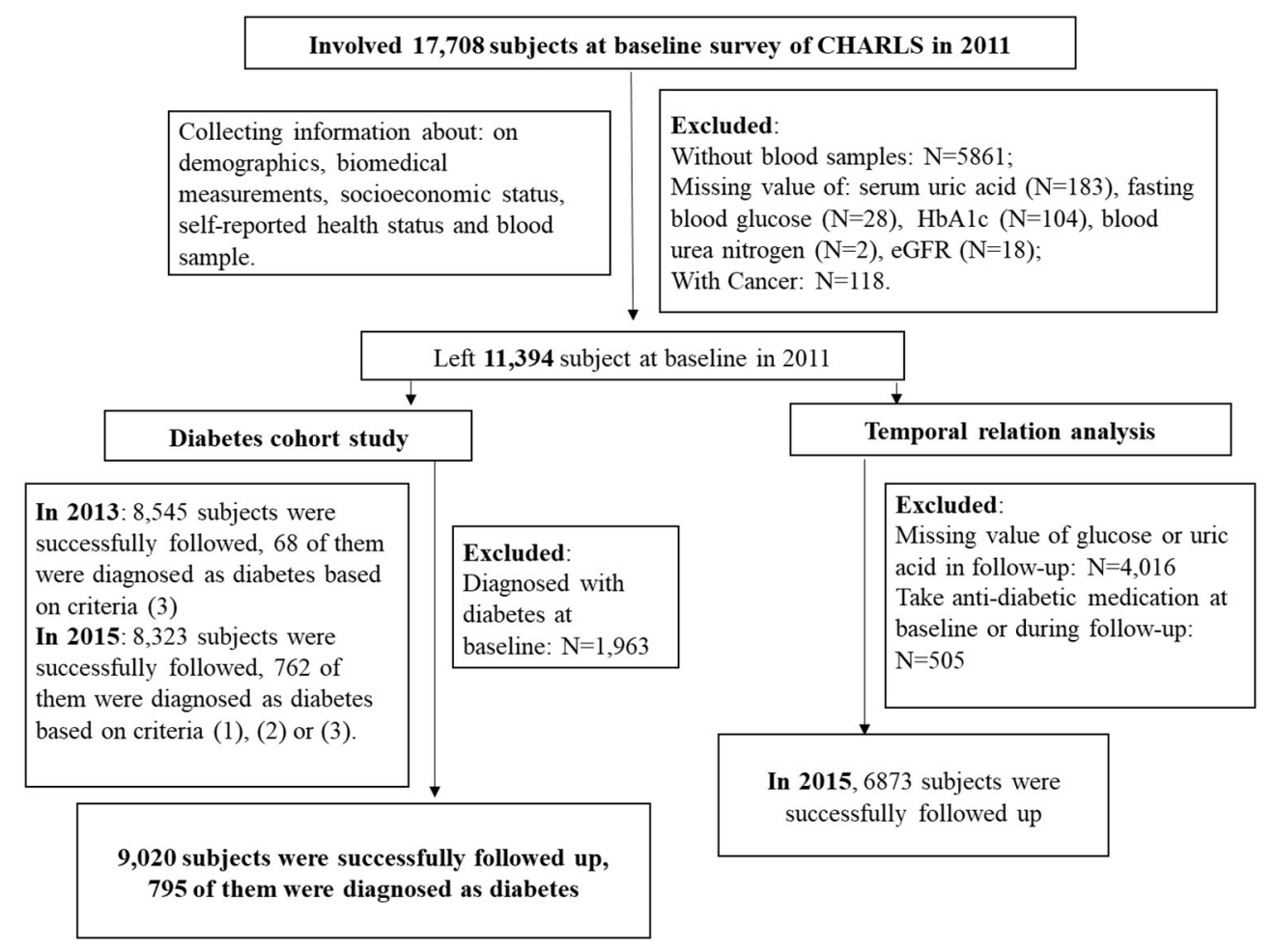

FIGURE 1 | Flow diagram of patients included in this study.

\section{Demographic, Anthropometric, and Biochemical Parameters}

According to the common classification in the existing literature, we divided the education level into the following four levels, including no formal education or illiterate, elementary or below, middle school, and high school or above. Based on the setting of the questionnaire, we simply divided marital status married or living with a partner, vs. others. Blood pressure was measured using an Omron HEM-7200 sphygmomanometer in the sitting position, three consecutive measurements were taken using the standard method, $45 \mathrm{~s}$ apart, and the average of the three results was taken as the final blood pressure. Hypertension means systolic blood pressure $\geq 140 \mathrm{mmHg}$ or diastolic blood pressure $\geq 90 \mathrm{mmHg}$, and we also classified people into the hypertension group who had been diagnosed with hypertension by their doctor or currently using antihypertensive medication. A higher level of high-sensitivity C-reactive protein (hs-CRP) over $3 \mathrm{mg} / \mathrm{dL}$ indicates inflammation in the body (23). The estimated eGFR was calculated using the Chronic Kidney Disease Epidemiology Collaboration equation (24).

\section{Definition of Primary Variables}

Participants who meet one of the following criteria are considered to have type 2 diabetes: (1) FPG $\geq 7.0 \mathrm{mmol} / \mathrm{L}(126 \mathrm{mg} / \mathrm{dL})$; (2) random plasma glucose (without overnight fasting) $\geq 11.1$ $\mathrm{mmol} / \mathrm{L}(200 \mathrm{mg} / \mathrm{dL})$; (3) $\mathrm{HbAlc} \geq 48 \mathrm{mmol} / \mathrm{mol}$ (6.5\%), (4) self-reported physician-diagnosed diabetes; and (5) currently taking antidiabetic medication.

The definition of hyperuricemia is different for men and women, for men with SUA $\geq 420 \mu \mathrm{mol} / \mathrm{L}$, and for women with SUA $\geq 360 \mu \mathrm{mol} / \mathrm{L}$ (25). We further divided participants into four groups using SUA gender-specific quartiles.

Once participants met one of the following criteria, they were diagnosed with dyslipidemia: (1) total cholesterol (TC) $\geq 240$ $\mathrm{mg} / \mathrm{dl}$, (2) high-density lipoprotein cholesterol (HDL-C) $<40$ $\mathrm{mg} / \mathrm{dl}$, (3) low-density lipoprotein cholesterol (LDL-C) $>160$ $\mathrm{mg} / \mathrm{dl}$, and (4) triglycerides (TG) $\geq 200 \mathrm{mg} / \mathrm{dl}$ (26).

Body mass index was calculated as dividing weight (kilogram) by height (meter) squared, and they were further divided into four categories (27), as follows: underweight (BMI $\left.<18.5 \mathrm{~kg} / \mathrm{m}^{2}\right)$, normal weight $\left(\mathrm{BMI}<24 \mathrm{~kg} / \mathrm{m}^{2}\right)$, overweight $\left(\mathrm{BMI}<28 \mathrm{~kg} / \mathrm{m}^{2}\right)$, and obese $\left(\mathrm{BMI} \geq 28 \mathrm{~kg} / \mathrm{m}^{2}\right)$.

\section{Statistical Analysis}

Statistical analysis was conducted by STATA version 16.0 (StataCorp, College Station, TX, USA) and R 4.0.3 (R Project for Statistical Computing), and a $p$-value $<0.05$ (2-tailed) was considered statistically significant. To make this study represent the overall level of middle-aged and elderly people in China, we took blood weight published in the CHARLS database in 2011 as the initial weight, further introducing the jackknife method to conduct weighted analysis based on the PPS sampling design. 
Baseline characteristics of study participants were reported by percentages for categorical variables or mean (standard error, SEM) for continuous variables. Groups were compared with one-way analysis of variance or the Kruskal-Wallis test for continuous variables, and Cochran-Mantel-Haenszel chi-square test for categorical variables.

Since new cases of diabetes were investigated at the 2013 or 2015 follow-up surveys, we were unable to estimate personyear accurately, in addition to the high incidence of diabetes in middle-aged and elderly people, prevented us from using odds ratios $(O R)$ calculated by logistic regression to estimate relative risk $(R R)$, since the use of $O R$ instead of $R R$ is artificially appropriate for rare events, Instead, we introduced the Cox proportional hazards regression with a robust variance estimator to estimate the $R R$, we set the follow-up time to 1 , and we used the Breslow method to break ties (28).

In addition, our previous study has confirmed an L-shape association between SUA and blood glucose at the same measurement point (19), but we did not know whether the relation of baseline SUA and the risk of diabetes during followup is linear. So, we introduced restricted cubic spline models to examine the dose-response association of SUA (continuous) with follow-up blood glucose (continuous). If the above doseresponse relationship was linear, we further conducted Cox proportional hazards regression to evaluate the association of SUA quartiles or continuous SUA levels (per $100 \mu \mathrm{mol} / \mathrm{L}$ elevations) or hyperuricemia (yes/no) with the risk of diabetes.

Further, the longitudinal changes of uric acid and blood glucose measured at two-time points are typically a crosslagged panel design (29). A regression residual analysis was initially used to identify the baseline and follow-up uric acid and blood glucose after adjusting all potential confounding factors, and then, values of adjusted residuals were standardized by Z-transformation (mean $=0$, standard deviation $=1$ ). The crosslagged path coefficients $\left(\rho_{1}\right.$ and $\left.\rho_{2}\right)$ were estimated simultaneously based on the correlation matrix using the maximum likelihood method in R 4.0.3 (Package: "lavaan"). The validity of model fitting was indicated by the root mean square residual (RMR) and comparative fitness index (CFI) (30), and $\mathrm{RMR}<0.05$ and CFI $>0.90$ indicate good fit to the observed data.

Finally, a Karlson-Holm-Breen (KHB) (31) method was constructed to examine the mediation effect of baseline BMI and dyslipidemia on the association between baseline SUA and follow-up risk of diabetes.

\section{RESULTS}

\section{The Characteristics Regarding the Study Variables}

Baseline demographic and clinical characteristics of the study population are given in Table 1. A total of 9,020 individuals (4,185 men and 4,835 women) were included in this study. At baseline, the mean BMI was $23.31 \mathrm{~kg} / \mathrm{m}^{2}$, the FPG level was $99.63 \mathrm{mg} / \mathrm{dl}$, the uric acid level was $270.05 \mu \mathrm{mol} / \mathrm{L}$, the HbA1c level was $5.08 \%$, and the prevalence of dyslipidemia was nearly
39.55\%. After 4 years of follow-up, BMI increased by 0.4 units and SUA levels increased by $20 \mathrm{umol} / \mathrm{L}$. A total of 795 participants were diagnosed with new-onset diabetes, with a cumulative 4year incidence of $8.81 \%$. Compared with women, men were more likely to be current smokers, regular drinkers, with higher hs-CRP, BUN, and SUA levels at baseline. In contrast, never smoking, never drinking, being illiterate, with higher BMI, and eGFR level were more common in women.

\section{Dose-Response Relation of Baseline Uric Acid and Incidence Diabetes}

As shown in Figure 2, the $\beta_{2}$ coefficient of non-linear trend calculated by restricted cubic spline was not significant, suggesting a linear dose-response association of SUA with blood glucose [FPG (Figure 2A) and HbAlc (Figure 2B) as continuous variables, diabetes (Figure 2C) as a dichotomous variable, respectively].

The linear coefficients between SUA and diabetes are shown in Table 2. In the total samples, compared with the lowest sexspecific quartile of SUA levels, individuals in the higher quartiles of SUA had a higher incident risk of diabetes. The RRs of incident diabetes were 1.00 (95\% CI: 0.82-1.23), 1.14 (95\% CI: 0.94-1.39), and 1.37 (95\% CI: 1.11-1.69) for individuals in Q2, Q3, and Q4, respectively ( $p$-trend $<0.01$; basic model). Additionally, per 100 $\mu \mathrm{mol} / \mathrm{L}$ of SUA level increase was significantly associated with 1.21 (95\% CI: 1.09-1.36)-fold higher incident risk of diabetes in the basic model. Additionally, those participants diagnosed with hyperuricemia at baseline had a $41 \%$ increased risk of developing diabetes during follow-up (95\% CI: 7-86\%; $p<$ 0.01 ). The observed association attenuated and was no longer significant after further adjustment for baseline BMI levels (basic model + BMI) or baseline dyslipidemia levels (basic model + dyslipidemia), whether for SUA as a continuous or categorical variable.

Stratified analysis by sex found that SUA was not significantly associated with the risk of diabetes in the men subgroup. Whereas, in women, the basic model is similar to the total population, compared with the first sex-specific quartile of SUA levels, the RRs of diabetes were 1.08 (95\% CI: $0.81-$ 1.44), 1.34 (95\% CI: 1.02-1.77), 1.64 (95\% CI: 1.24-2.18) for quartile $2-4$ ( $p$-trend $=0.001$, basic model), respectively. A 100 $\mu \mathrm{mol} / \mathrm{L}$ increment of SUA was linked with $40 \%$ (95\% CI: $21-$ $62 \%$ ) elevated risk of diabetes in women (basic model). The observed association decreased but remained significant after further adjustment for BMI or baseline dyslipidemia levels in women (basic model + BMI: $p$-trend $<0.01$; basic model + dyslipidemia: $p$-trend $<0.01)$. For women, we further stratified by menopause status. As shown in Table 3, after adjusting for confounders, the relation of baseline SUA and the risk of diabetes in premenopausal women were not statistically significant. In the postmenopausal female subgroup, the results were similar to the overall analysis of all female patients, no matter took uric acid as a continuous variable or dichotomic variable, and the increase of uric acid can lead to the increased risk of diabetes in postmenopausal women. 
TABLE 1 | Weighted characteristics of the study participants according to different gender.

\begin{tabular}{|c|c|c|c|c|}
\hline & All & Men & Women & $p$-value \\
\hline \multicolumn{5}{|l|}{ Baseline (2011) } \\
\hline$n$ & 9,020 & 4,185 & 4,835 & \\
\hline Married or living with a partner (\%) & 82.74 & 86.80 & 78.77 & $<0.001^{\dagger}$ \\
\hline Smoking status (\%) & & & & $<0.001^{\dagger}$ \\
\hline Current smoker & 28.08 & 54.08 & 5.21 & \\
\hline Alcohol consumption (\%) & & & & $<0.001^{\dagger}$ \\
\hline Never drinking & 58.71 & 59.31 & 83.90 & \\
\hline Former drinkers & 7.20 & 7.26 & 3.79 & \\
\hline Occasional drinkers & 8.61 & 8.69 & 5.64 & \\
\hline Elementary or below & 39.51 & 43.62 & 35.76 & \\
\hline Middle school & 22.49 & 27.98 & 17.48 & \\
\hline High school or above & 13.27 & 16.44 & 10.38 & \\
\hline Dyslipidemia (\%) & 39.55 & 40.37 & 38.79 & $0.38^{\dagger}$ \\
\hline High hs-CRP (\%) & 17.50 & 19.58 & 15.60 & $<0.01^{\dagger}$ \\
\hline Hypertension (\%) & 41.84 & 40.86 & 42.72 & $0.18^{\dagger}$ \\
\hline Age (years) & $58.59(0.21)$ & $59.16(0.27)$ & $58.07(0.24)$ & $<0.001^{\S}$ \\
\hline $\mathrm{BMI}\left(\mathrm{kg} / \mathrm{m}^{2}\right)$ & $23.31(0.07)$ & $22.86(0.08)$ & $23.74(0.06)$ & $<0.001^{\S}$ \\
\hline Systolic (mmHg) & $130.83(0.36)$ & $130.88(0.43)$ & $130.77(0.36)$ & $0.46^{\ddagger}$ \\
\hline Diastolic (mmHg) & $75.64(0.22)$ & $76.13(0.31)$ & $75.20(0.24)$ & $<0.001^{\S}$ \\
\hline WC (cm) & $83.81(0.24)$ & $83.63(0.31)$ & $83.97(0.23)$ & $<0.01^{\S}$ \\
\hline FPG (mg/dl) & $99.63(0.23)$ & $99.64(0.30)$ & $99.62(0.25)$ & $0.65^{\S}$ \\
\hline HbA1c (\%) & $5.08(0.02)$ & $5.08(0.01)$ & $5.07(0.01)$ & $0.55^{\ddagger}$ \\
\hline \multicolumn{5}{|l|}{ Follow-up (2015) } \\
\hline $\mathrm{BMI}\left(\mathrm{kg} / \mathrm{m}^{2}\right)$ & $23.76(0.05)$ & $23.21(0.06)$ & $24.26(0.06)$ & $<0.001^{\S}$ \\
\hline SUA ( $\mu \mathrm{mol} / \mathrm{L})$ & $298.11(1.47)$ & $331.94(2.10)$ & $268.57(1.46)$ & $<0.001^{\S}$ \\
\hline FPG (mg/dl) & $98.13(0.35)$ & $98.73(0.50)$ & $97.61(0.44)$ & $0.83^{\S}$ \\
\hline HbA1c (\%) & $5.81(0.01)$ & $5.78(0.01)$ & $5.84(0.01)$ & $<0.001^{\S}$ \\
\hline Diabetes (\%) & 8.81 & 8.48 & 9.10 & $0.30^{\dagger}$ \\
\hline
\end{tabular}

Unless indicated otherwise, data are given as the means (SEM) or as percentages.

${ }^{\dagger}$ Cochran-Mantel-Haenszel chi-square test.

$\ddagger$ One-way analysis of variance (ANOVA).

$\S$ The Kruskal-Wallis test.

\section{The Crosslagged Path Analysis of Uric Acid and Blood Glucose}

As shown in Figure 3A, in all pathways, only the path coefficients $\left(\rho_{1}\right)$ from baseline SUA to follow-up blood glucose $\left(\rho_{1}=0.24\right.$, $p=0.03$ ) were significant in the total population after adjusting for confounding factors, and the RMR and CFI were 0.009 and 0.998 for FPG, 0.002 and 0.999 for $\mathrm{HbAlc}$, respectively, indicating a good fit to the observed data, suggesting that SUA was more likely to affect blood glucose and is a risk factor for diabetes. Further stratified by sex, we found that this path coefficient between baseline uric acid and follow-up blood glucose was significant only in the female subgroup (for 
A

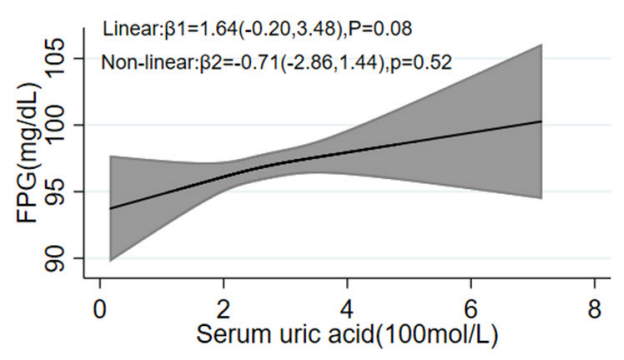

C

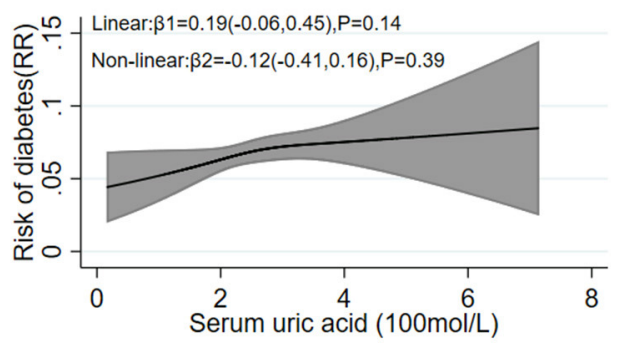

B

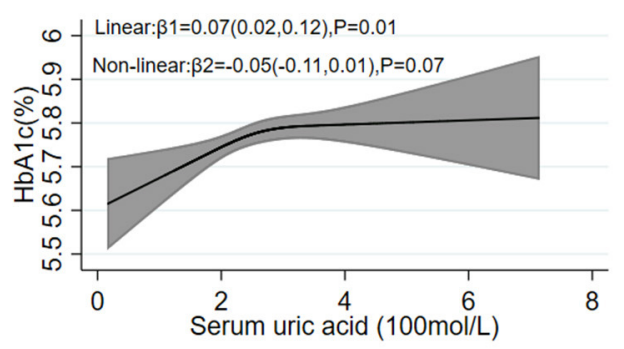

FIGURE 2 | Restricted cubic spline analysis between baseline SUA levels and follow-up FPG (A), HbA1c (B), and the risk of diabetes (C). FPG, fasting plasma glucose; HbA1c, hemoglobin A1c.

FPG: $\rho_{1}=0.05, p<0.01$; for HbA1c: $\rho_{1}=0.04, p=0.03$; Figure $3 \mathrm{C}$ ), and no longer had a statistical association in men (Figure 3B).

\section{The Mediation Analysis in the Women Subgroup}

Since the above statistical analysis confirmed that SUA was an independent risk factor for the development of diabetes in women in terms of both the strength of association $(R R)$ and the temporal sequence (crosslagged path analysis), we analyzed the mediating effects of BMI and dyslipidemia in the female subgroup, respectively (Figure 4). After adjusting for all potential confounding factors in Figure 4A, the increased level of baseline SUA was positively correlated with baseline BMI $\left(\beta_{1}=0.12, p<0.001\right)$ or dyslipidemia $\left(\beta_{1}=0.07\right.$, $p<0.001$ ), and the change in baseline BMI or dyslipidemia could significantly increase the incidence of diabetes (for BMI: $\beta_{2}=0.38, p<0.001$; for dyslipidemia: $\beta_{2}=0.57$, $p<0.001)$. Similar results were found in a subgroup of postmenopausal women who were assessed separately for BMI and dyslipidemia (Figure 4B). Multiple parallel mediation analyses (Table 4) showed a significant total effect of baseline SUA on diabetes risk $(\beta=0.19,95 \% C I: 0.08-0.29, p<$ $0.001)$, the direct effect of SUA on the relation of diabetes accounted for $58.13 \%$ ( $\beta=0.11,95 \%$ CI: $0.01-0.22, p<0.001)$ of the total effect, and the combined indirect effect of BMI and dyslipidemia explained residual 41.78\% $(\beta=0.8,95 \%$ CI: $0.06-0.10, p<0.001)$ of the total effect. Additionally, in the stratified analysis, we confirmed this mediating effect in postmenopausal women.

\section{DISCUSSION}

This study represents a comprehensive examination of the longitudinal relationship between uric acid concentration and diabetes in a national-based population. Our results help reconcile conflicting evidence in the literature and demonstrate the following: (1) an unidirectional relationship between SUA and blood glucose was identified, and increased SUA is an independent risk factor for diabetes; (2) In men, the longitudinal association between SUA and diabetes was not significant after adjustment for confounders.; (3) In women, SUA is an important risk factor for the development of diabetes, especially in postmenopausal women, and this harmful effect of uric acid is partly mediated by BMI and dyslipidemia.

Since the sex-specific cutoff points for hyperuricemia have been widely used globally, it is of importance to identify whether there are sex-specific associations between SUA and diabetes. Previous studies reported that SUA increment was associated with increased risk for diabetes in women but not in men $(32,33)$. Consistent with this, we confirmed that for every 100 $\mu \mathrm{mol} / \mathrm{L}$ increase in SUA in women, the risk of diabetes was 1.31 -fold higher in women. Not only in its association with diabetes but also gender have differences also often been seen in other fields. In the Framingham Heart Study, levels of SUA were associated with an increased risk of cardiovascular death in women but not in men (34). Additionally, Ndrepepa et al. 
TABLE 2 | Risk ratios (95\% confidence intervals) of diabetes according to SUA.

\begin{tabular}{|c|c|c|c|c|}
\hline & $\begin{array}{l}\text { Univariate } \\
R R(95 \% \text { Cl) }\end{array}$ & $\begin{array}{l}\text { Basic model } \\
R R(95 \% C l)\end{array}$ & $\begin{array}{l}\text { Basic model + BMI } \\
R R(95 \% \mathrm{Cl})\end{array}$ & $\begin{array}{l}\text { Basic model + Dyslipidemia } \\
R R(95 \% \mathrm{Cl})\end{array}$ \\
\hline \multicolumn{5}{|l|}{ Total participants } \\
\hline Q1 & Ref. & Ref. & Ref. & Ref. \\
\hline Q2 & $1.01(0.83,1.23)$ & $1.00(0.82,1.23)$ & $0.96(0.79,1.18)$ & $0.99(0.81,1.21)$ \\
\hline Q3 & $1.16(0.95,1.40)$ & $1.14(0.94,1.39)$ & $1.08(0.89,1.31)$ & $1.10(0.90,1.34)$ \\
\hline Normal uric acid & Ref. & Ref. & Ref. & \\
\hline Hyperuricemia & $1.50(1.16,1.93)^{\star \star}$ & $1.41(1.07,1.86)^{*}$ & $1.32(0.99,1.74)$ & $1.33(1.01,1.75)^{\star}$ \\
\hline Uric acid (100 umol/l) & $1.17(1.08,1.27)^{\star \star \star}$ & $1.21(1.09,1.36)^{\star \star}$ & $1.15(0.03,1.29)^{\star}$ & $1.17(1.05,1.30)^{\star}$ \\
\hline \multicolumn{5}{|l|}{ Male participants } \\
\hline Q1 & Ref. & Ref. & Ref. & Ref. \\
\hline$P$ for trend ${ }^{a}$ & 0.882 & 0.559 & 0.748 & 0.729 \\
\hline Normal uric acid & Ref. & Ref. & Ref. & Ref. \\
\hline Hyperuricemia & $1.43(1.01,2.04)^{\star}$ & $1.42(0.99,2.01)$ & $1.32(0.99,1.74)$ & $1.41(0.97,2.16)$ \\
\hline Uric acid (100 umol/l) & $1.05(0.91,1.21)$ & $1.07(0.90,1.26)$ & $1.04(0.88,1.23)$ & $1.05(0.89,1.24)$ \\
\hline \multicolumn{5}{|l|}{ Female participants } \\
\hline Q1 & Ref. & Ref. & Ref. & Ref. \\
\hline Q2 & $1.09(0.82,1.45)$ & $1.08(0.81,1.44)$ & $1.05(0.79,1.40)$ & $1.08(0.81,1.44)$ \\
\hline Q3 & $1.40(1.07,1.83)^{\star}$ & $1.34(1.02,1.77)^{\star}$ & $1.25(0.95,1.65)$ & $1.27(0.97,1.67)$ \\
\hline Q4 & $1.81(1.40,2.34)^{\star \star \star}$ & $1.64(1.24,2.18)^{\star \star}$ & $1.45(1.09,1.93)^{\star}$ & $1.49(1.13,1.97)^{\star}$ \\
\hline$P$ for trend ${ }^{a}$ & $<0.001$ & 0.001 & 0.006 & 0.001 \\
\hline Normal uric acid & Ref. & Ref. & Ref. & Ref. \\
\hline
\end{tabular}

${ }^{*} p<0.05$.

${ }^{*} p<0.01$.

${ }_{* \star *} p<0.001$.

The based model was adjusted for age (standardized), gender, education levels, marital status and place of residence, high-sensitivity C-reactive protein, blood urea nitrogen, smoking status, drinking status, and hypertension.

${ }^{a}$ Test for linear trend was performed using the median SUA levels for each quartile as a continuous variable.

SUA, serum uric acid; Q1, Q2, Q3, and Q4 were the quartiles of SUA; BMI, body mass index.

also demonstrated that hyperuricemia could predict an increased cardiovascular risk of mortality in both genders, with a stronger association in women (35). Possible mechanisms for gender differences include the following: first, there were differences in BMI, hypertension, WC, BUN, eGFR, and blood lipid between genders, which partly explained the different reactivities of uric acid and blood glucose in men and women; second, a reduction in estrogen levels after menopause in women may result in dysregulation of blood glucose and lipid metabolism (36), and of the 4,835 women included in this study, about $68 \%$ were postmenopausal, and our subgroup analysis proved that elevated uric acid increased the risk of diabetes only in postmenopausal women. Besides, a genome-wide association study reported a significant association between the SLC2A9 and urate concentrations, whereas the proportion of the variance of SUA concentrations explained by expression levels was $3.5 \%$ in men and $15 \%$ in women (37), which possibly suggests a genetic basis for the sex differences.

The mediating effect is based on the premise that the causal relationship between independent and dependent variables holds. Previous studies have identified uric acid as a risk factor for diabetes $(18,19,38)$; however, Lu et al. used an animal model which confirmed that hyperuricemia could accelerate but do not cause diabetes by inhibiting islet $\beta$-cell survival (39); only male mice were used in their study, which may impede the extrapolation from animal experiments to human populations. Meanwhile, several Mendelian randomized (4042) studies also do not support a causal role of SUA for the development of diabetes and limit the expectation that UAlowering drugs will be effective in the prevention of diabetes (39); whereas the genetic risk score in above Mendelian randomization studies only explained $2.9 \%$ of SUA variation, the strength 
TABLE 3 | Risk ratios (95\% confidence intervals) of diabetes according to SUA in women subgroup analysis.

\begin{tabular}{|c|c|c|c|c|}
\hline & $\begin{array}{l}\text { Univariate } \\
R R(95 \% C l)\end{array}$ & $\begin{array}{l}\text { Basic model } \\
R R(95 \% C l)\end{array}$ & $\begin{array}{l}\text { Basic model + BMI } \\
R R(95 \% C l)\end{array}$ & $\begin{array}{l}\text { Basic model + Dyslipidemia } \\
R R(95 \% C l)\end{array}$ \\
\hline \multicolumn{5}{|c|}{ Without menopause $(N=1,545)$} \\
\hline Q1 & Ref. & Ref. & Ref. & Ref. \\
\hline Q2 & $1.16(0.67,2.03)$ & $1.16(0.66,2.03)$ & $1.14(0.65,2.00)$ & $1.15(0.66,2.02)$ \\
\hline Q3 & $1.51(0.88,2.58)$ & $1.40(0.80,2.44)$ & $1.35(0.78,2.33)$ & $1.31(0.75,2.29)$ \\
\hline Q4 & $2.20(1.34,3.61)^{\star \star}$ & $1.44(0.83,2.50)$ & $1.30(0.75,2.62)$ & $1.28(0.73,2.22)^{\star}$ \\
\hline$P$ for trend ${ }^{a}$ & $<0.01$ & 0.557 & 0.698 & 0.700 \\
\hline Normal uric acid & Ref. & Ref. & Ref. & Ref. \\
\hline Hyperuricemia & $1.66(0.71,3.85)$ & $1.38(0.56,3.41)$ & $1.29(0.52,3.20)$ & $1.27(0.51,3.15)$ \\
\hline Uric acid (100 umol/l) & $1.57(1.25,1.99)^{\star \star \star}$ & $1.25(0.95,1.65)$ & $1.18(0.89,1.57)$ & $1.16(0.88,1.54)$ \\
\hline \multicolumn{5}{|c|}{ Menopause $(N=3,290)$} \\
\hline Q1 & Ref. & Ref. & Ref. & Ref. \\
\hline Q2 & $1.05(0.75,1.47)$ & $1.04(0.74,1.47)$ & $1.00(0.72,1.42)$ & $1.05(0.75,1.48)$ \\
\hline Q3 & $1.31(0.96,1.79)$ & $1.28(0.93,1.76)$ & $1.18(0.85,1.62)$ & $1.23(0.89,1.69)$ \\
\hline Q4 & $1.64(1.22,2.21)^{\star \star}$ & $1.57(1.15,2.16)^{\star \star}$ & $1.39(1.01,1.93)^{\star}$ & $1.44(1.05,1.97)^{\star}$ \\
\hline$P$ for trend $^{\mathrm{a}}$ & $<0.01$ & 0.02 & 0.02 & 0.04 \\
\hline Normal uric acid & Ref. & Ref. & Ref. & Ref. \\
\hline Hyperuricemia & $1.55(1.03,2.37)^{\star}$ & $1.21(0.76,1.92)$ & $1.08(0.67,1.75)$ & $1.11(0.71,1.73)$ \\
\hline Uric acid (100 umol/I) & $1.40(1.21,1.62)^{\star \star \star}$ & $1.35(1.15,1.58)^{\star \star \star}$ & $1.26(1.07,1.49)^{\star \star}$ & $1.28(1.10,1.49)^{\star \star}$ \\
\hline
\end{tabular}

${ }^{*} p<0.05$

${ }^{*} p<0.01$

${ }^{* \star *} p<0.001$.

The based model was adjusted for age (standardized), gender, education levels, marital status and place of residence, high-sensitivity C-reactive protein, blood urea nitrogen, smoking status, drinking status, and hypertension.

${ }^{a}$ Test for linear trend was performed using the median SUA levels for each quartile as a continuous variable.

SUA, serum uric acid; Q1, Q2, Q3, Q4 were the quartiles of SUA; BMI, body mass index.

TABLE 4 | Multiple parallel mediation effect of baseline BMI and dyslipidemia on the relation of baseline SUA and risk of diabetes in women.

\begin{tabular}{|c|c|c|c|c|c|c|}
\hline & Mediators & Effect & SE & $95 \% \mathrm{Cl}$ & $P$ & Proportion \\
\hline \multicolumn{7}{|c|}{ Total women $(N=4,835)$} \\
\hline Direct effect & & 0.11 & 0.05 & $(0.01,0.22)$ & $<0.001$ & $58.13 \%$ \\
\hline \multirow[t]{3}{*}{ Indirect effect } & BMI+ Dyslipidemia & 0.08 & 0.01 & $(0.06,0.10)$ & $<0.001$ & $41.87 \%$ \\
\hline & BMI & 0.04 & 0.01 & $(0.03,0.07)$ & $<0.001$ & $23.05 \%$ \\
\hline & Dyslipidemia & 0.04 & 0.01 & $(0.02,0.06)$ & $<0.001$ & $18.82 \%$ \\
\hline Direct effect & & 0.12 & 0.06 & $(-0.01,0.23)$ & 0.06 & $60.68 \%$ \\
\hline \multirow[t]{3}{*}{ Indirect effect } & BMI+ Dyslipidemia & 0.07 & 0.01 & $(0.05,0.10)$ & $<0.001$ & $39.32 \%$ \\
\hline & $\mathrm{BMl}$ & 0.04 & 0.01 & $(0.03,0.07)$ & $<0.001$ & $23.05 \%$ \\
\hline & Dyslipidemia & 0.03 & 0.01 & $(0.02,0.06)$ & $<0.001$ & $18.82 \%$ \\
\hline Total effect & & 0.19 & 0.06 & $(0.07,0.31)$ & $<0.001$ & $100 \%$ \\
\hline
\end{tabular}

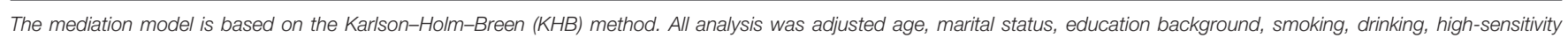
C-reactive protein, and blood urea nitrogen.

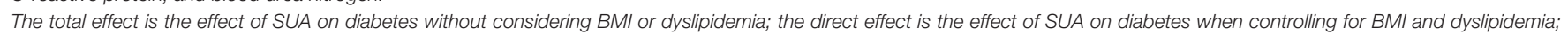
the indirect effect is the effect of SUA on diabetes through BMI or dyslipidemia; proportion: mediation effect by BMI or dyslipidemia is calculated by indirect effect/total effect $\times 100$.

of the evidence may be insufficient. Taking the above results into consideration, we further explored this chicken-and-egg question by crosslagged path analysis, which is a powerful statistical approach in dissecting a causal relationship between intercorrelated variables (29). In our results, the path coefficient from baseline SUA to follow-up blood glucose was statistically significant in the total population and the female subgroup, providing statistical evidence that uric acid is a possible cause of blood glucose; therefore, we only explore the potential mediation effect in women.

Similar to our findings, some research pointed out that the association between SUA and the risk of diabetes is largely 


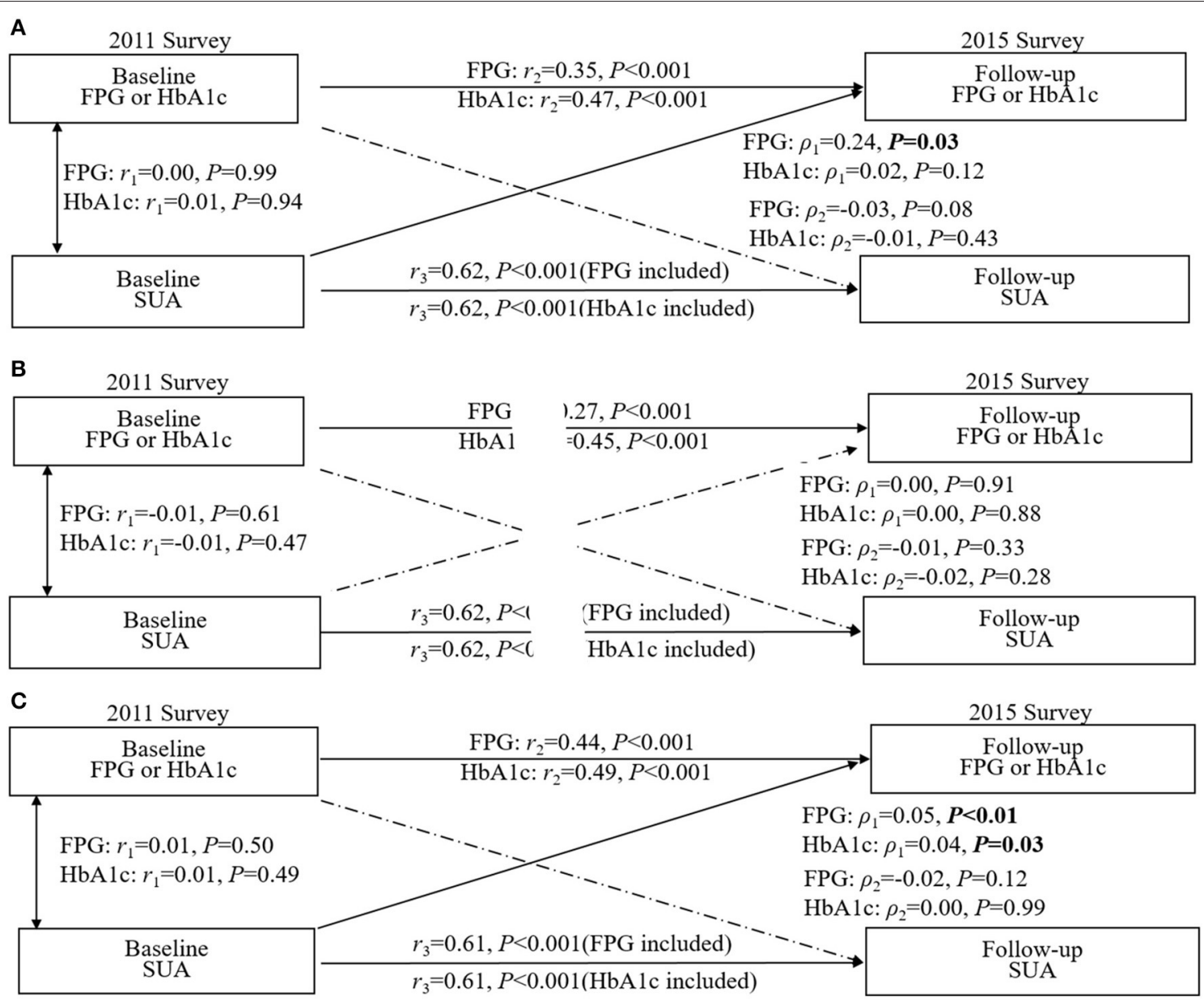

FIGURE 3 | Crosslagged path analysis models for the association of fasting plasma glucose (FPG) and HbA1c with SUA. (A) included all participants ( $n=6,873)$; (B) included male participants $(n=3,162)$; $(\mathbf{C})$ included female participants $(n=3,711)$. All results were adjusted for age, sex, marital status, education background, waist circumference, smoking, drinking, hypertension and high-sensitivity C-reactive protein. $\rho_{1}$, crosslagged path coefficient from baseline SUA to follow-up blood glucose (FPG and $\mathrm{HbA} 1 \mathrm{C}$ ); $\rho_{2}$, crosslagged path coefficient from baseline blood glucose (FPG and HbA1C) to follow-up SUA. $r_{1}$, represents synchronous correlations; $r_{2}$ and $r_{3}$ represent tracking correlations.

decreased or eliminated after adjusting for BMI $(10,11)$. UA can affect adipocytes by inducing upregulation of proinflammatory factors and downregulation of the insulin sensitizer and antiinflammatory factor adiponectin (43). Adiponectin is negatively associated with BMI and body fat (44) since low levels of adiponectin are associated with the development of insulin resistance (45), and it could be speculated that adiponectin is part of the link between UA and insulin resistance (46). Additionally, data coming from the National Health and Nutrition Examination Survey (NHANES) demonstrated a significant association between elevated SUA levels and the increased prevalence of abdominal obesity, hypertriglyceridemia, and hyperglycemia (47), and some prospective studies also showed that elevated SUA levels may increase the risk of hypertriglyceridemia (48). Hypertriglyceridemia is known as a dominant lipid abnormality in insulin resistance by inducing elevated levels of free fatty acids, which plays an important role in the development of diabetes (49). The interactive effects of increased TG and the LDL-C/HDL-C ratio suggest that dyslipidemia might exaggerate the risk of diabetes in hypertensive patients (50). This study demonstrated that nearly $42 \%$ total effect of UA on diabetes is mediated by BMI and dyslipidemia in women. This provides a theoretical basis for the designation of preventive measures. For middle-aged and older women, especially those with higher uric acid levels, reasonable diet control and physical exercise can keep them in the normal $\mathrm{BMI}$ range and reduce the probability of dyslipidemia, which can help us avoid developing diabetes in the future.

Strengths of this study include a wealth of sociodemographic, clinical, and laboratory variables collected by medical professionals in a standardized manner with a low rate of missing data. In addition, the confirmation of multiple mediating roles of BMI and dyslipidemia helps to clarify the pathogenetic pathway of uric acid to diabetes. What is more, CHARLS adopts probability proportional to size sampling, so the results can represent the current level of China and have 


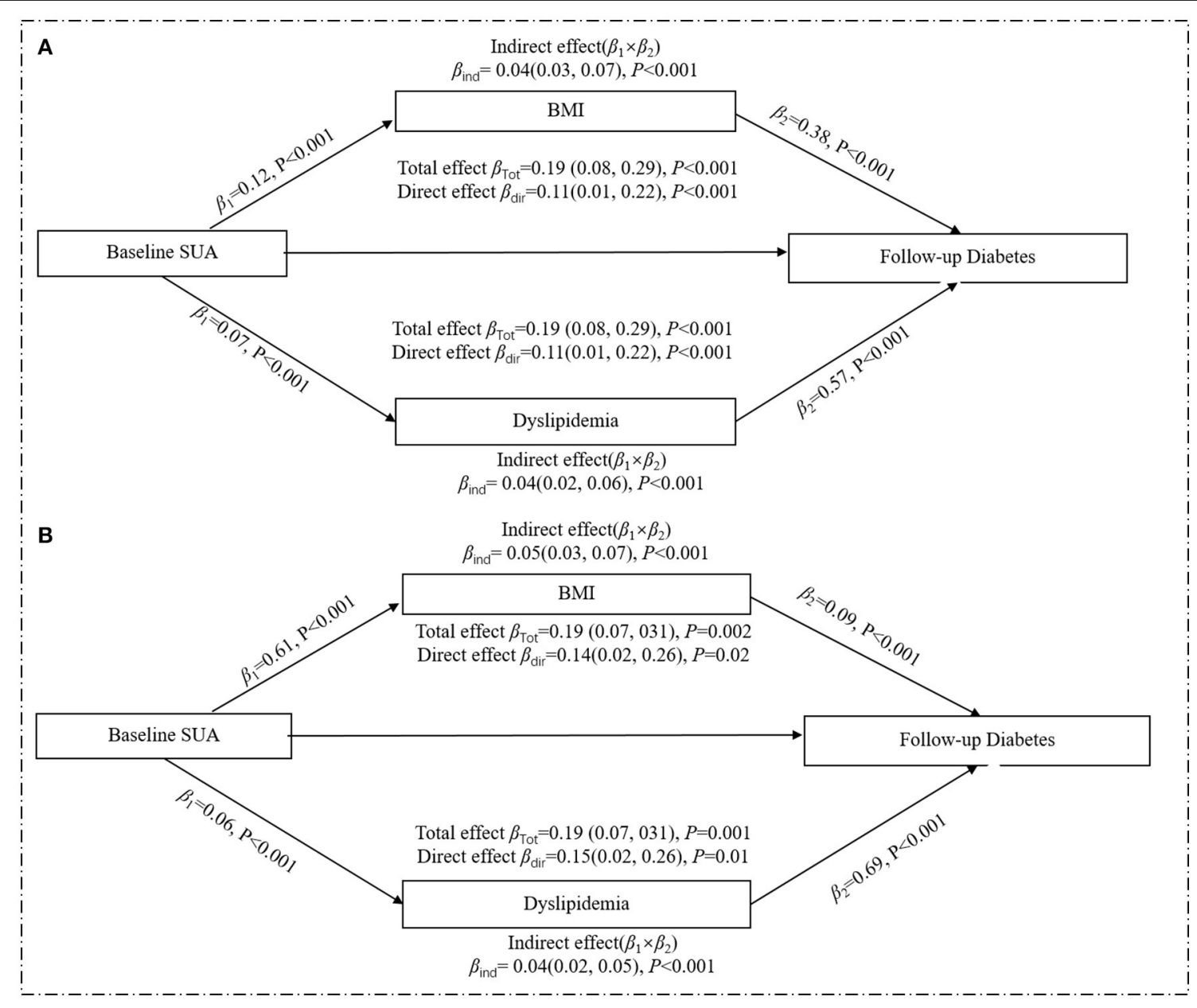

FIGURE 4 | Mediation effect of baseline obesity on the relation of baseline SUA and risk of diabetes in the women. (A) included all women ( $n=4,835) ;(\mathbf{B})$ included the postmenopausal female subgroup $(n=3,290)$. All analyses were adjusted age, marital status, education background, smoking, drinking, high-sensitivity C-reactive protein, menopausal status, and eGFR. The total effect is the effect of SUA on diabetes without considering BMI or dyslipidemia; the direct effect is the effect of SUA on diabetes when controlling for BMI and dyslipidemia; the indirect effect is the effect of SUA on diabetes through BMI or dyslipidemia. Mediation effects by BMI or dyslipidemia are calculated by indirect effect/total effect $\times 100$. $\beta$, regression coefficients.

good extrapolation. Study weaknesses include our follow-up period which was only 4 years, which was comparatively shorter than other cohort studies, and despite adjustment for a range of potential confounders, the possibility of residual and unmeasured confounders may not be ruled out, such as diet, drugs, and genetic information. Besides, as in any observation study, causality cannot be determined by the strength of relation and temporal relation alone, and more definitive basic studies are warranted to confirm causality between UA and diabetes.

\section{CONCLUSION}

In conclusion, using a sample drawn from the China Health and Retirement Longitudinal Study, this study extended the findings of the previous literature by confirming that SUA and risk of type 2 diabetes are only significant in middle-aged and elderly Chinese women, and further quantified the mediating proportion of BMI and dyslipidemia in the relationship between SUA and risk of type 2 diabetes in women. For middle-aged and elderly Chinese women, especially those with high uric acid, in addition to corresponding measures to reduce uric acid, integrally targeted interventions and strategies that can alleviate BMI and dyslipidemia should be combined to reduce the risk of diabetes.

\section{DATA AVAILABILITY STATEMENT}

The datasets presented in this study can be found in online repositories. The names of the repository/repositories and accession number(s) can be found at: the CHARLS data can be freely downloaded from the official website (http://charls.pku. edu.cn/index/zh-cn.html). 


\section{ETHICS STATEMENT}

The studies involving human participants were reviewed and approved by the CHARLS is a large-scale interdisciplinary research project sponsored by the National Development Institute of Peking University. Ethical approval for all the CHARLS waves, therefore, was granted from the Institutional Review Board (IRB) at Peking University, including anthropometrics (IRB00001052-11015) and biomarker collection (IRB00001052-11014). The patients/participants provided their written informed consent to participate in this study.

\section{AUTHOR CONTRIBUTIONS}

HJ designed the study. FC performed the statistical analyses, drafted the manuscript, and created the figures. YL, HZ, and LT interpreted the data and edited the manuscript. All authors listed have approved the manuscript that is enclosed and

\section{REFERENCES}

1. Saeedi P, Petersohn I, Salpea P, Malanda B, Karuranga S, Unwin N, et al. Global and regional diabetes prevalence estimates for 2019 and projections for 2030 and 2045: results from the international diabetes federation diabetes atlas, 9(th) edition. Diabetes Res Clin Pract. (2019) 157:107843. doi: 10.1016/j.diabres.2019.107843

2. Yang T, Chu CH, Bai CH, You SL, Chou YC, Chou WY, et al. Uric acid level as a risk marker for metabolic syndrome: a Chinese cohort study. Atherosclerosis. (2012) 220:525-31. doi: 10.1016/j.atherosclerosis.2011.11.014

3. Dehghan A, van Hoek M, Sijbrands E, Hofman A, Witteman J. High serum uric acid as a novel risk factor for type 2 diabetes. Diabetes Care. (2008) 31:361-2. doi: 10.2337/dc07-1276

4. Juraschek SP, McAdams-Demarco M, Miller ER, Gelber AC, Maynard JW, Pankow JS, et al. Temporal relationship between uric acid concentration and risk of diabetes in a community-based study population. Am J Epidemiol. (2014) 179:684-91. doi: 10.1093/aje/kwt320

5. Taniguchi Y, Hayashi T, Tsumura K, Endo G, Fujii S, Okada K. Serum uric acid and the risk for hypertension and type 2 diabetes in Japanese men: The Osaka Health Survey. J Hypertens. (2001) 19:120915. doi: 10.1097/00004872-200107000-00005

6. Kodama S, Saito K, Yachi Y, Asumi M, Sugawara A, Totsuka K, et al. Association between serum uric acid and development of type 2 diabetes. Diabetes Care. (2009) 32:1737-42. doi: 10.2337/dc09-0288

7. Chen MY, Zhao CC, Li TT, Zhu Y, Yu TP, Bao YQ, et al. Serum uric acid levels are associated with obesity but not cardio-cerebrovascular events in Chinese inpatients with type 2 diabetes. Sci Rep. (2017) 7:40009. doi: 10.1038/srep40009

8. Chu FY, Chang CC, Huang PH, Lin YN, Ku PW, Sun JT, et al. The association of uric acid calculi with obesity, prediabetes, type 2 diabetes mellitus, and hypertension. Biomed Res Int. (2017) 2017:7523960. doi: 10.1155/2017/7523960

9. Frühbeck G, Toplak H, Woodward E, Yumuk V, Maislos M, Oppert JM. Obesity: the gateway to ill health - an EASO position statement on a rising public health, clinical and scientific challenge in Europe. Obes Facts. (2013) 6:117-20. doi: 10.1159/000350627

10. Norvik JV, Storhaug HM, Ytrehus K, Jenssen TG, Zykova SN, Eriksen $\mathrm{BO}$, et al. Overweight modifies the longitudinal association between uric acid and some components of the metabolic syndrome: The Tromsø Study. BMC Cardiovasc Disord. (2016) 16:85. doi: 10.1186/s12872-0160265-8

11. Ferrara LA, Wang H, Umans JG, Franceschini N, Jolly S, Lee ET, et al. Serum uric acid does not predict incident metabolic syndrome in a population with the corresponding author would take final responsibility for the paper.

\section{FUNDING}

This project was supported by the Research Development Fund of The Second Hospital of Shandong University (Grant No. 11681808), Construction of Intelligent Cloud Platform for Clinical Medicine Teaching based on multidisciplinary typical cases (Grant No. 2019Z10), and Jinan Clinical Medical Science and Technology innovation plan (Grant No. 202019194).

\section{ACKNOWLEDGMENTS}

The authors are grateful to the China Health and Retirement Longitudinal Study (CHARLS) research and field team for collecting the data and providing the data. high prevalence of obesity. Nutr Metabol Cardiovasc Dis. (2014) 24:13604. doi: 10.1016/j.numecd.2014.06.002

12. Johnson R, Lanaspa M, Gaucher E. Uric acid: a danger signal from the RNA world that may have a role in the epidemic of obesity, metabolic syndrome, and cardiorenal disease: evolutionary considerations. Semin Nephrol. (2011) 31:394-9. doi: 10.1016/j.semnephrol.2011.08.002

13. American Diabetes Association. Standards of medical care in diabetes-2012. Diabetes Care. (2012) 35:S11-63. doi: 10.2337/dc12-s011

14. Liang Z, Qiu Q, Wu J, Zhou J, Xu T, Zhang M, et al. Alcohol drinking, dyslipidemia, and diabetes: a population-based prospective cohort study among inner mongolians in china. Biomed Environ Sci. (2016) 29:55562. doi: $10.3967 /$ bes 2016.074

15. PapeŽíková I, Pekarová $\mathrm{M}$, Kolárová $\mathrm{H}$, Klinke $\mathrm{A}$, Lau D, Baldus $\mathrm{S}$, et al. Uric acid modulates vascular endothelial function through the down regulation of nitric oxide production. Free Radic Res. (2013) 47:828. doi: $10.3109 / 10715762.2012 .747677$

16. Sautin YY, Nakagawa T, Zharikov S, Johnson RJ. Adverse effects of the classic antioxidant uric acid in adipocytes: NADPH oxidase-mediated oxidative/nitrosative stress. Am J Physiol Cell Physiol. (2007) 293:C58496. doi: 10.1152/ajpcell.00600.2006

17. Maples KR, Mason RP. Free radical metabolite of uric acid. J Biol Chem. (1988) 263:1709-12. doi: 10.1016/S0021-9258(19)77933-2

18. Wang Y, Chi J, Che K, Chen Y, Sun X, Wang Y, et al. Fasting plasma glucose and serum uric acid levels in a general Chinese population with normal glucose tolerance: a U-shaped curve. PLoS ONE. (2017) 12:e0180111. doi: 10.1371/journal.pone.0180111

19. Cheng F, Yin X, Duan W, Ye R, Zhu Y, Jia C. Different-shaped curves for serum uric acid with and without diabetes: results from China Health and Retirement Longitudinal Study. J Diabetes. (2019) 11:4319. doi: 10.1111/1753-0407.12863

20. Rodríguez G, Soriano LC, Choi HK. Impact of diabetes against the future risk of developing gout. Ann Rheum Dis. (2010) 69:2090-4. doi: 10.1136/ard.2010.130013

21. Zhao Y, Hu Y, Smith JP, Strauss J, Yang G. Cohort profile: the China Health and Retirement Longitudinal Study (CHARLS). Int J Epidemiol. (2014) 43:618. doi: 10.1093/ije/dys203

22. Chen X, Crimmins E, Hu PP, Kim JK, Meng Q, Strauss J, et al. Venous blood-based biomarkers in the china health and retirement longitudinal study: rationale, design, and results from the 2015 wave. Am J Epidemiol. (2019) 188:1871-7. doi: 10.1093/aje/kwz170

23. Yan S, Li J, Li S, Zhang B, Du S, Gordon-Larsen P, et al. The expanding burden of cardiometabolic risk in China: the China Health and Nutrition Survey. Obes Rev. (2012) 13:810-21. doi: 10.1111/j.1467-789X.2012.01016.x 
24. Levey AS, Stevens LA, Schmid CH, Zhang YL, Castro AF, 3rd, Feldman $\mathrm{HI}$, et al. A new equation to estimate glomerular filtration rate. Ann Intern Med. (2009) 150:604-12. doi: 10.7326/0003-4819-150-9-20090505000006

25. Zhu Y, Pandya BJ, Choi HK. Prevalence of gout and hyperuricemia in the US general population: the National Health and Nutrition Examination Survey 2007-2008. Arthritis Rheum. (2011) 63:3136-41. doi: 10.1002/art.30520

26. Mazzeo S, Cervelli R, Elisei R, Tarantini G, Cappelli C, Molinaro E, et al. mRECIST criteria to assess recurrent thyroid carcinoma treatment response after radiofrequency ablation: a prospective study. J Endocrinol Invest. (2018) 41:1389-99. doi: 10.1007/s40618-018-0886-0

27. Alberti KG, Zimmet P, Shaw J. The metabolic syndrome-a new worldwide definition. Lancet. (2005) 366:1059-62. doi: 10.1016/S0140-6736(05)67402-8

28. Cummings P. Methods for estimating adjusted risk ratios. Stata J. (2009) 9:175-96. doi: 10.1177/1536867X0900900201

29. Kenny D. Cross-lagged panel correlation: a test for spuriousness. Psychol Bull. (1975) 82:887-903. doi: 10.1037/0033-2909.82.6.887

30. Chen W, Li S, Fernandez C, Sun D, Lai CC, Zhang T, et al. Temporal relationship between elevated blood pressure and arterial stiffening among middle-aged black and white adults: the Bogalusa Heart Study. Am J Epidemiol. (2016) 183:599-608. doi: 10.1093/aje/kwv274

31. Kohler U, Karlson KB, Holm A. Comparing coefficients of nested nonlinear probability models. Stata J. (2011) 11:42038. doi: 10.1177/1536867X1101100306

32. Shani M, Vinker S, Dinour D, Leiba M, Twig G, Holtzman EJ, et al. High normal uric acid levels are associated with an increased risk of diabetes in lean, normoglycemic healthy women. J Clin Endocrinol Metab. (2016) 101:3772-8. doi: 10.1210/jc.2016-2107

33. Choi B, Kim D, Baek M, Ryu Y, Kim S, Lee M, et al. Hyperuricaemia and development of type 2 diabetes mellitus in Asian population. Clin Exp Pharmacol Physiol. (2018) 45:499-506. doi: 10.1111/1440-1681.12911

34. Culleton BF, Larson MG, Kannel WB, Levy D. Serum uric acid and risk for cardiovascular disease and death: the Framingham Heart Study. Ann Intern Med. (1999) 131:7-13. doi: 10.7326/0003-4819-131-1-199907060-00003

35. Ndrepepa G, Cassese S, Braun S, Fusaro M, King L, Tada T, et al. A genderspecific analysis of association between hyperuricaemia and cardiovascular events in patients with coronary artery disease. Nutr Metabol Cardiovasc Dis. (2013) 23:1195-201. doi: 10.1016/j.numecd.2013.03.005

36. Yahyaoui R, Esteva I, Haro-Mora JJ, Almaraz MC, Morcillo S, Rojo-Martínez $\mathrm{G}$, et al. Effect of long-term administration of cross-sex hormone therapy on serum and urinary uric acid in transsexual persons. J Clin Endocrinol Metab. (2008) 93:2230-3. doi: 10.1210/jc.2007-2467

37. Döring A, Gieger C, Mehta D, Gohlke H, Prokisch H, Coassin S, et al. SLC2A9 influences uric acid concentrations with pronounced sex-specific effects. Nat Genet. (2008) 40:430-6. doi: 10.1038/ng.107

38. Choi HK, Ford ES. Haemoglobin Alc, fasting glucose, serum C-peptide and insulin resistance in relation to serum uric acid levels-the Third National Health and Nutrition Examination Survey. Rheumatology. (2008) 47:7137. doi: 10.1093/rheumatology/ken066

39. Lu J, He Y, Cui L, Xing X, Liu Z, Li X, et al. Hyperuricemia predisposes to the onset of diabetes via promoting pancreatic $\beta$-cell death in uricase-deficient male mice. Diabetes. (2020) 69:1149-63. doi: 10.2337/db19-0704

40. Keerman M, Yang F, Hu H, Wang J, Wang F, Li Z, et al. Mendelian randomization study of serum uric acid levels and diabetes risk: evidence from the Dongfeng-Tongji cohort. BMJ Open
Diabetes Res Care. (2020) 8:e000834. doi: 10.1136/bmjdrc-2019-0 00834

41. Sluijs I, Holmes MV, van der Schouw YT, Beulens JW, Asselbergs FW, Huerta JM, et al. A mendelian randomization study of circulating uric acid and type 2 diabetes. Diabetes. (2015) 64:3028-36. doi: 10.2337/db14-0742

42. Pfister R, Barnes D, Luben R, Forouhi NG, Bochud M, Khaw KT, et al. No evidence for a causal link between uric acid and type 2 diabetes: a Mendelian randomisation approach. Diabetologia. (2011) 54:25619. doi: 10.1007/s00125-011-2235-0

43. Baldwin W, McRae S, Marek G, Wymer D, Pannu V, Baylis C, et al. Hyperuricemia as a mediator of the proinflammatory endocrine imbalance in the adipose tissue in a murine model of the metabolic syndrome. Diabetes. (2011) 60:1258-69. doi: 10.2337/db10-0916

44. Matsubara M, Maruoka S, Katayose S. Inverse relationship between plasma adiponectin and leptin concentrations in normal-weight and obese women. Eur J Endocrinol. (2002) 147:173-80. doi: 10.1530/eje.0.1470173

45. Yamamoto Y, Hirose H, Saito I, Nishikai K, Saruta T. Adiponectin, an adipocyte-derived protein, predicts future insulin resistance: two-year followup study in Japanese population. J Clin Endocrinol Metab. (2004) 89:8790. doi: 10.1210/jc.2003-031163

46. Wu L, Parhofer KG. Diabetic dyslipidemia. Metabol Clin Exp. (2014) 63:146979. doi: 10.1016/j.metabol.2014.08.010

47. Ford E, Li C, Cook S, Choi HJC. Serum concentrations of uric acid and the metabolic syndrome among US children and adolescents. Circulation. (2007) 115:2526-32. doi: 10.1161/CIRCULATIONAHA.106.657627

48. Kuwabara M, Borghi C, Cicero A, Hisatome I, Niwa K, Ohno M, et al. Elevated serum uric acid increases risks for developing high LDL cholesterol and hypertriglyceridemia: a five-year cohort study in Japan. Int J Cardiol. (2018) 261:183-8. doi: 10.1016/j.ijcard.2018.03.045

49. Zhu X, Deng F, Lei SF. Meta-analysis of atherogenic index of plasma and other lipid parameters in relation to risk of type 2 diabetes mellitus. Prim Care Diabetes. (2015) 9:60-7. doi: 10.1016/j.pcd.2014.03.007

50. Hong M, Ling Y, Lu Z, Liu Y, Gu P, Shao J, et al. Contribution and interaction of the low-density lipoprotein cholesterol to high-density lipoprotein cholesterol ratio and triglyceride to diabetes in hypertensive patients: a cross-sectional study. J Diabetes Investig. (2019) 10:131-8. doi: 10.1111/jdi.12856

Conflict of Interest: The authors declare that the research was conducted in the absence of any commercial or financial relationships that could be construed as a potential conflict of interest.

Publisher's Note: All claims expressed in this article are solely those of the authors and do not necessarily represent those of their affiliated organizations, or those of the publisher, the editors and the reviewers. Any product that may be evaluated in this article, or claim that may be made by its manufacturer, is not guaranteed or endorsed by the publisher.

Copyright (c) 2022 Cheng, Li, Zheng, Tian and Jia. This is an open-access article distributed under the terms of the Creative Commons Attribution License (CC BY). The use, distribution or reproduction in other forums is permitted, provided the original author(s) and the copyright owner(s) are credited and that the original publication in this journal is cited, in accordance with accepted academic practice. No use, distribution or reproduction is permitted which does not comply with these terms. 